Archives de sciences sociales des religions

172 | octobre-décembre

Bulletin Bibliographique

\title{
Après bien des voyages
}

Jacques Le Brun

\section{(2) OpenEdition}

Journals

Édition électronique

URL : http://journals.openedition.org/assr/27225

DOI : $10.4000 /$ assr. 27225

ISSN : $1777-5825$

Éditeur

Éditions de l'EHESS

Édition imprimée

Date de publication : 1 octobre 2015

Pagination : 89-98

ISBN : 978-2-7132-2515-4

ISSN : 0335-5985

Référence électronique

Jacques Le Brun, «Après bien des voyages », Archives de sciences sociales des religions [En ligne], 172 I octobre-décembre, mis en ligne le 26 avril 2018, consulté le 03 mai 2019. URL : http:// journals.openedition.org/assr/27225 ; DOI : 10.4000/assr.27225 


\title{
Jacques Le Brun
}

\section{Après bien des voyages}

\author{
«Le poème de Jean de la Croix \\ s'achèvera comme on meurt, \\ après bien des voyages" \\ (La Fable mystique II, p. 128)
}

Publié dans la "Bibliothèque des histoires ", La Fable mystique est un livre d' " histoire ", c'est-à-dire un livre qui se soumet, et revendique cette soumission, aux procédures et à la légalité de la démarche historienne. Ainsi l'objet du travail, soit la mystique, la spiritualité, ou ce que recouvrent ces mots, possède une " historicité ", donc doit être étudié comme les autres objets historiques, même si c'est selon des modalités particulières, et même si, consciente d'elle-même, cette histoire ne cesse, parallèlement à son travail, de s'interroger sur ses conditions de possibilité et de réalisation, même si, en d'autres mots, elle possède aussi sa propre historicité.

Ainsi, au point de départ, il y a des « documents » (p. 23), mais ces documents ne sont pas le fait brut, immédiat, que l'on pourrait isoler (par exemple les " phénomènes mystiques ", l' " état mystique ", l' " expérience mystique »), mais sont «les effets d'activités sociales» (p. 23), par exemple des textes autobiographiques, des témoignages de contemporains, des biographies, des élaborations spirituelles à partir de ce qui a été dit ou observé, des textes de controverses théologiques, de dénonciations, de condamnations, etc. Nous devons tirer des caractères très particuliers de cette histoire un certain nombre de conséquences qui orientent tout travail portant sur ce qu'on appelle une "expérience » : «Il n'y a pas à proprement parler d'expérience individuelle. C'est là un mirage ", écrit Michel de Certeau (p. 166) à propos de Jean de la Croix et de Surin; ce qu'on appelle " expérience spirituelle » se trouve en quelque sorte " aliéné » dans le langage (p.166), est " postulé » par le travail historique qui, tout en sachant que c'est postulat et présupposé du travail, fait " comme si », et cela pour pouvoir opérer. Si l'expérience n'est que "là où elle se dit» (p. 166), Michel de Certeau peut poser, contre toute tentative, ou tentation, pour objectiver ou saisir une expérience primitive antérieure à son expression, et encore une fois à partir des écrits de Surin, à partir de sa "Science expérimentale », que "l'écriture n'est 
pas extrinsèque à l'expérience comme si elle la décrivait du dehors. Elle en "fait partie". Elle est elle-même "science expérimentale" " (p. 245).

La métaphore théâtrale s'impose en plusieurs des chapitres de La Fable mystique II : si l'écrit "donne corps au sujet ", s'il est " une voix qui en escompte une autre» (p. 245), il met « en scène » l'instance énonciatrice (p. 315), comme il est dit à propos de la lettre de Pascal à Mlle de Roannez; ou, comme le montre le texte inaugural de La Fable mystique II, " chaque texte ou chaque document (puisque nous sommes obligés de travailler sur eux) constitue un théâtre qu'organisent le lexique et la syntaxe d'un moment de l'histoire, mais où se marquent, comme en un corps touché, des actions singulières » (p. 50), un théâtre qui peut être souvent "théâtre de la cruauté » (p. 27). En un mot, que Michel de Certeau reprend à un commentateur contemporain de Racine, "les textes, pas plus que les objets d'art, ne peuvent être lus [...], ce sont des objets» (p.311), ce qui signifie qu'ils ne sont pas la voie d'accès à une réalité qui leur serait antérieure, qui existerait (mais où ?) avant que ces textes, ces documents, ne lui donnent « corps".

Toute tentative pour faire de la mystique l'accès à un monde autre ou essentiel se trouve, par cette conviction fondamentale, renvoyée à l'insignifiance, mais derechef un travail, une approche qui serait découverte d'un " objet », se trouvent à la fois justifiés et caractérisés.

Faire l'histoire de la mystique, dessiner les traits de cette "science étrange » qu'est la "science mystique " (p. 21), ne sera donc pas établir et analyser des "propositions ", un système de "vérités ". La variété des travaux consacrés depuis deux siècles à ces "documents » est là pour dissiper l'illusion d'une "science » qui serait «stable» (p. 38) : sociale et individuelle, la mystique pour ainsi dire «bouge» (p. 43).

Pour aller au centre de ce que montre le travail de Michel de Certeau, c'est moins un ensemble de dits qu'un dire, et moins des messages écrits qu'une écriture, qui en seront l'objet. C'est un des axes majeurs de La Fable mystique II. Le fait, d'ailleurs, ne nous surprendra pas si nous donnons son sens propre à ce terme de "fable » qui en est le titre, non pas le sens de ce qui est "fabuleux ", mais le sens étymologique : fari en latin (phêmi en grec) c'est "parler "; fabula c'est d'abord la conversation (donc une parole avec une adresse et l'attente ou la réception d'une réponse, une parole donc qui suppose de l'autre), fabula est conversation, et ensuite le sujet ou l'objet de la conversation, d'où le récit, dialogué ou mis en scène, et enfin, secondairement, un récit fictif ou mensonger; renvoyons ici à Ernout-Meillet.

En tout cas, la portée du titre donné au livre de 1982 était déjà dans la contribution de Michel de Certeau au débat qui l'opposa à Jean-Marie Domenach en 1973, publié dans Le christianisme éclaté en 1974, lorsqu'il posait, à propos des «textes » chrétiens, que « le travail chrétien », pendant deux mille 
ans, avait produit la «conversion de la légalité du texte [les Écritures d'Israël], c'est-à-dire ce qui lui restait de force, en la faiblesse d'une fable ». ${ }^{1}$ Et il dégageait le caractère, d'ordre "poétique ", de la fable : "La fable reste toujours dans la distance, comme l'autre poétique de l'effectivité historique, comme une utopie qui n'est articulée sur les topographies sociales que par un risque privé » (ibid., p. 98). Poésie et utopie, pour reprendre les signifiants de 1973, marquent ce qui des discours, spirituel, mystique, historique, etc., est de l'ordre du dire, au-delà ou en deçà des dits c'est-à-dire des vérités.

Cette sorte de décrochage entre le dire et ce qui est dit apparaît, de façon qui peut sembler paradoxale, dès le chapitre consacré par La Fable mystique II à Nicolas de Cues. Il y est, certes, question du regard, mais le commentaire de la préface du De Icona articule, et ancre pour ainsi dire, le regard sur un dire, anéantissant toute possibilité d'une position fixe ou surplombante, toute position assurée par une objectivité : "Fondamentalement, le regard est un dire qui organise tout l'espace. "Ton regard parle”, commente Nicolas de Cues. Il est énonciatif» (p. 90). «Le regard parle », écrit le cardinal, il parle non pour apporter une réponse "objective", mais pour introduire la question de l'autre, la question "Que me veut l'autre?». Et Michel de Certeau peut poser que ce qui est ici pertinent n'est pas un "ce qu'il veut dire », mais un dire qui induit un croire à la parole, qui est question de l'autre. Ce n'est que secondairement que se fera le "transfert [...] du "semblant", qui affectait le théâtre du regard, à la "vérité" que doit dire le discours » (p.92); et ici encore la métaphore du théâtre s'est imposée, non pour affecter d'irréalité cette « vérité " mais pour mettre en priorité cette construction, cet « acte " qui précède son objet, un " acte de croire " logiquement antérieur, mais contemporain dans son immédiateté, à «ce qu'il faut croire et comment » (p. 92).

Ce que nous apprend le montage cusain de la scène du regard, et qui est placé non seulement en tête du De Icona du cardinal, mais aussi dans les débuts de La Fable mystique II (comme La Fable mystique I s'ouvrait sur deux « lieux pour se perdre ", les monastères du Désert et le "Paradis » de Jérôme Bosch), ce que nous apprend ce montage donne le ton du livre où à chaque pas la question du dire, d'une parole qui est acte, prime sur le dit, que ce dernier soit philosophique, théologique, historique, scientifique, psychanalytique, etc.

Ce que met en scène le montage théâtral du De Icona, Michel de Certeau le fait jouer à propos de Jean de la Croix : au départ non pas un message, non pas des vérités, même philosophiques (s'écartant ici, p. 128, de Jean Baruzi dont il admirait néanmoins le travail), mais un «cri ", signe d'une absence : "Où t'estu caché, Aimé ? ( p. 125), une parole " sans raison " au sens de " sans motifs »

1. Michel de Certeau, Jean-Marie Domenach, Le christianisme éclaté, Paris, Le Seuil, 1974, p. 97. 
(ohne warum est tenté de dire le lecteur d'Angelus Silesius), sans rien qui "n'autorise », " ni l'ordre d'un signifié, ni le référentiel d'une réalité »(p. 126). Pour Jean de la Croix, il n’y a ni sens ni texte "révélé » (p. 130) et la Parole s'écoute dans son "énoncé " (ibid.). D’où les versions différentes du Cantico et pas un «bon» texte, l'unité n'est qu'esprit ou parole. Ici encore le dire est "plus» que le dit (p. 130). Le Prologue du Cantico de Jean de la Croix ouvre non au sens mais à la question même, inaugurale, de la possibilité d'un dire : "Peut-on dire?" (p 142). Ce qui se constitue c'est un "réseau interlocutoire ", un "conversar» (p. 144), où ne se révèle pas un "savoir ", pas un "discours du “savoir” " (ce n'est "pas ça ", " ni ça »), mais la possibilité de "la parole du “croire” " (p. 144), une foi qui postule qu' «il y a " de l'autre, une foi à laquelle l'acte poétique donne un «lieu» (p. 136). Il faudra revenir sur cette dimension, fondamentale au sens où elle fait fondement, au sens de fondation, de l'acte.

Déjà en 1972, Michel de Certeau, dans une table ronde avec Maurice Bellet, André Dumas et d'autres ${ }^{2}$, posait que quelque chose parle avant nous et cernait les conditions d' " un acte d'écrire dont le texte présente les traces dans son organisation même ", ce qui permettait de déceler dans l'énoncé une énonciation, dans ce qui est dit l'acte de dire, voyant dans "la démarche sémiotique " une possibilité de le découvrir (ibid., p. 48).

Cependant, nous l'avons noté à la suite de Michel de Certeau, l'historien se trouve devant des textes, des mots, seules traces de cette parole initiale et instauratrice, seules traces du dire derrière les dits. Or c'est dans le texte même, dans ces unités que sont les "mots " (ainsi Surin devant le texte de Jean de la Croix, p. 188-189), que l'historien, comme le spirituel du XVII ${ }^{\mathrm{e}}$ siècle, découvre des traces de la parole. Ainsi se révèle l'aspect « littéraire » de toute histoire de la mystique, "littéraire " mais d'une tout autre façon que cette « histoire littéraire » qu'avait jadis élaborée l'abbé Bremond. Certes ce dernier avait eu conscience que c'est dans des textes, dans ce qui était devenu « littérature spirituelle » que s'exprimait et se retrouvait ce qu'on appelle l' «expérience » spirituelle; mais, et certes avec subtilité, il s'en tenait, faute d'ancrage théorique (philosophique, linguistique, sémiotique, etc.), aux discours que la culture de son temps, l' « histoire littéraire » et sa propre culture théologique lui suggéraient, et cela même si la psychologie (un Delacroix, etc.), la psychiatrie (un Janet, etc.), la philosophie alliée à la science des textes (un Baruzi, etc.) auraient déjà pu lui ouvrir d'autres horizons.

Chez Michel de Certeau au contraire, on est orienté plutôt vers la littérarité que vers la littérature, plutôt vers le signifiant, la lettre, que vers le sens exprimé par le mot. À la fois la linguistique, la sémiotique et la psychanalyse lacanienne

2. Maurice Bellet et al., Crise du biblisme, chance de la Bible, Paris, Epi, 1973, p. 46. 
sont passées par là. Le mot, peut-on dire, "parle ", c'est ce que pose Michel de Certeau à partir des fragments de Jean de la Croix : le mot "parle, c'est-à-dire qu'il y a de l'autre en lui » (p. 157), il est « offert à toute une série de circulations » (ibid.), ce sont des « éclats » (p. 149), un réseau adressé à tous, au pluriel, pluriel qui « est la forme du “dire” »(p. 155), qui exprime l'échange verbal avant l'écrit, des « dichos» (p. 154).

Il s'agit d'un usage de la langue qui en écoute ce qui peut s'entendre et non se comprendre, en un mot une "petite musique » (p. 162); une musique, une inaccessibilité sémantique, " un inexprimable par rapport à l'énoncé », une poésie présente dans les textes étudiés, mais aussi dans le style d'écriture de l'historien ; peut-être, nous y reviendrons, y a-t-il là ce que l'adjectif « mystique » suggère de cette parole, cette "fable».

Ce dire, en tant que tel, est marqué, pour ainsi dire marqué par sa négation : à la fois un dire et un ne pas dire, comme l'exprime La Vive Flamme de Jean de la Croix : "Âmes, dites-le. Oh non, ne le dites pas" (p. 185). Le dire implique ainsi en soi toute la positivité féconde d'une négation qui n'est pas pure et simple absence mais possibilité d'une fondation impliquant altérité et perte, négation qui travaille toujours ce que nous appelons "mystique ».

Cette pratique de la négation, dans les écrits mystiques, mais aussi dans les élaborations théoriques de Michel de Certeau, lecteur de longtemps attentif de Hegel et de la Phénoménologie de l'esprit, est un des éléments qui donnent à ce travail et à cette écriture de l'histoire sa dimension proprement philosophique dont il conviendrait d'étudier les origines et les conséquences. La remarque de Luce Giard en 1991 selon laquelle Hegel fut, avec Freud, le «principal interlocuteur intellectuel » de Michel de Certeau, doit être prise dans toute son importance $^{3}$.

Une des formes ou des manifestations de cette active négativité pourrait être ce qu'à maintes reprises, et déjà dans l'entretien de 1973, Michel de Certeau désignait comme l'altérité, c'est-à-dire "rapport à l'autre " ${ }^{4}$, mais aussi "blessure du discours" (ibid., p. 51), altérité qui fait trace dans le "théâtre » du corps sous la forme du "pâtir " (auquel est consacré le chapitre 6 de La Fable mystique II) et dans l'écrit sous celle de motions, de modalités, de la possibilité d'une énonciation.

Des figures incarnent cette primauté du dire, les unes figures de la tradition, les autres figures de l'expérience; elles l'incarnent, c'est-à-dire lui donnent un corps ou font d'un corps pour ainsi dire le porte-voix d'une parole antérieure au message et à sa vérité ; d'une part l'ange, d'autre part la glossolalie. L'une

3. Luce Giard éd., Histoire, mystique et politique, Michel de Certeau, Grenoble, Jérôme Millon, 1991, p. 133.

4. Michel de Certeau, Jean-Marie Domenach, Le christianisme éclaté, op. cit., p. 37. 
et l'autre de ces figures, qui se glissaient déjà dans La Fable mystique I, ainsi l'ange entrevu à Paris par Mme Guyon, donnent à ce volume La Fable mystique II, parmi les textes les plus inspirés que Certeau ait écrits. L'histoire de l'ange en Occident s'articule en effet sur ces deux polarités de l'être et du dire : l'ange est un dire, un parler, celui de Dieu, il n'a pas d'être propre, il prend la forme du passage, il est le passage ("zébrure dans l'ordre cosmique ", écrit Michel de Certeau p. 260); on le définit comme "adjectif ", " angélique ", qui, comme l'adjectif "mystique ", sert à déterminer toute réalité, discours, corps, etc. (p. 263) : est " angélique " ce qui me touche, à "l'instant ", hors du temps et dans le temps, simplement signe que ce dire qui me frappe vient de l'autre. Serait-il le nom, nom non essentiel mais « adjectif », d'une modalité antérieure à tout «être" ? On comprend l'importance de ce chapitre dans l'économie du livre et on mesure, il faudra y revenir, comment la modalité joue dans la pensée et l'écriture (des mystiques et peut-être de leur historien) un rôle déterminant. L'ange, qui pour l'historien de la spiritualité classique pourrait apparaître comme l'objet marginal, voire folklorique, des croyances et des dévotions du fidèle, devient au contraire comme la figure, figure "théorique " (au sens étymologique de ce mot), figure rare et passante, de la pure parole prenant apparence d'un corps, un dire devenant apparence personnelle (si cette alliance oxymorique de mots peut se penser), un être pour ainsi dire postérieur à cette parole, un être qui est parole mais qui touche, blesse et stigmatise comme l'ange de la transverbération thérésienne. Qu'ensuite la théologie, avec Denys l'Aréopagite et saint Thomas, comme le montre Michel de Certeau (p. 266-267), attribue un « être » à l'ange, un être antérieur à ce dire ou à ce toucher, ne fait que révéler, par l'attribution de cette forme personnelle, la difficulté à penser le dire décroché de ce qui est dit et de qui dit.

L'autre figure de ce dire décroché de l'être est apportée par les glossolalies, " opéra du dire » (p. 337). Là encore, nous sommes sur les marges de ce qui est reconnu socialement et qui est objet de l'histoire. Mais ce n'est pas par curiosité pour le folklore ou attrait pour les formes "populaires " de la religion ou de la religiosité, comme ce fut souvent le cas il y a une quarantaine d'années, que Michel de Certeau donne aux glossolalies une place d'enjeu décisif dans son travail. Leur importance est de donner un exemple, pour ainsi dire expérimental, d'un « dire premier » (p. 338), d'une langue qui n'est pas une langue, d'une «pure fable (fari, parler) ", dire à la fois exceptionnel (aux frontières de l'étrange et du pathologique) et intime présence à la marge de toute parole, de toute " conversation ordinaire ", des "bruits d'autre » (p. 339). Là se trouvent en radical décrochement dire et ce qui est dit, et peut-être en deçà même du dire, le "vouloir dire " et "un "vouloir dire quelque chose" » (p. 344), à la fois avant le dire et ce qui du dire tombe comme une chute (au sens artisanal) de ce dire, un vouloir et un reste, des débris. 
Déjà en mettant un "vouloir ", un "vouloir dire " en position inaugurale par rapport à un dire et à ce qui est dit, nous avons mis le doigt sur un caractère de cet objet qui a pu recevoir la détermination de l'adjectif «mystique ». L'objet du travail de Michel de Certeau sera non pas l'étude de doctrines, d'idées, dogmes ou vérités, qu'ils soient théologiques ou historiques, mais celle d'une « origine » au sens d'un commencement, d'une invention, d'une instauration, et aussi d'altérations et de modalités. Car il s'agit aussi d'un "acte ", il ne s'agit pas de ce qui préexisterait; il s'agit du surgissement d'un discours. Il y a là un objet qui oblige à déplacer les formes et les pratiques de l'histoire.

Comme le faisait Nicolas de Cues, dont la figure en tête de La Fable mystique II est, je crois, tout à fait significative à la fois comme objet d'un travail et exemple d'une pratique, valant pour tout travail, c'est se placer « à la source de chaque doctrine », c'est susciter "l'instauration de nouvelles unités » (p. 113); que cette origine soit pensée par le cusain comme «don d'en haut» (p. 101102), c'est sous la forme d'un "principe ", au sens étymologique de ce mot, surgissement premier, que l'expérience inaugurale se manifeste.

De cette origine l'expérience poétique offre un bon exemple, objet constant du travail de Michel de Certeau, la poésie de Jean de la Croix, mais aussi celle de Hölderlin (p. 126, 128) ou celle de tel poète contemporain comme René Char (p. 112) ou au tome I de La Fable mystique Catherine Pozzi. La poésie apparaît comme « éveilleuse » (p. 133), elle est « commencement» (p. 124), origine d'une "genèse " (p. 133).

Étudier de ce point de vue, du point de vue du geste instaurateur, poétique et fondateur, les textes témoignages de l'expérience dite mystique implique de pratiquer une nouvelle sorte d'histoire qui, sans rien sacrifier de la rigueur et de la légalité de la discipline historique, rende compte de ce qui, suscitant une histoire, n'est lisible que par une autre lecture des documents et qui s'exprime par une écriture elle-même poétique, c'est-à-dire créatrice, capable d'en rendre compte. L'attention à la poésie, aux "bruits énonciatifs ", plus qu'à la prose des textes et aux énoncés (p. 228), l'attention aux "débris inducteurs de textes doctrinaux» (p. 228), à l' "indistinct» et au «confus» (p. 220), implique le surgissement de documents peu pratiqués jusqu'ici par l'historien et aussi un autre usage des documents, une autre façon d'écrire l'histoire, un nouveau style. L'exemple du livre et de la lecture en serait un exemple : contrairement à l'usage le plus communément admis, selon lequel la lecture permettrait de connaître faits, vérités et leçons, dans la "lecture absolue », que pratiquent les spirituels et dont ils transmettent l'expérience, le livre est un objet dont le contenu n'épuise pas la force ; il fait « commencement », «c'est un signifiant de l'Autre » (p. 206), il est l'index d' « un autre vouloir-dire » (p. 206). Mais si pour le spirituel le livre et la lecture ont cette fonction, la pratique de l'historien s'en trouve altérée : luimême, dans les textes qui sont ses sources, il va devoir guetter ces «commencements d'histoire» (p. 228) qui généreront chez lui un autre mode d'écriture. 
De cette pratique et de cette écriture, les deux tomes de La Fable mystique nous donnent un exemple peut-être pas réitérable. On est frappé par ce qui pourrait n'apparaître que comme un trait de méthode : un texte, plus ou moins long, est donné de façon inaugurale et ensuite ce texte suscite une écriture qui n'est pas une simple explication ou un commentaire, mais une écriture homologue à ce que ces pages inaugurales font lire, des commencements, des appels, la surrection d'autres travaux. Ainsi la préface du De Icona de Nicolas de Cues, le prologue du Cantico de Jean de la Croix, la IV lettre de Pascal à Mlle de Roannez dans le tome II, mais déjà au tome I une page de l'Histoire lausiaque ou une autre de Mme Guyon ou un poème de Catherine Pozzi. Mais déjà dans le tome I une nouvelle lecture, celle de l'image, le Jardin des Délices de Jérôme Bosch, était à l'œuvre, non plus d'un texte supposé contenir un message, mais une image suscitant une nouvelle écriture en accord avec ce que cette image fait surgir chez l'historien; et on pourrait y ajouter l'image, trop connue pour être reproduite mais insistante dans l'esprit du lecteur, la transverbération de sainte Thérèse suscitant au tome II de nouvelles pages à l'historien. À chaque fois, des écrits (ou une œuvre d'art, tableau ou sculpture), avec des procédés qui peuvent être différents (ainsi les schémas qui rendent lisible l'architecture de la lettre de Pascal, p. 316-318), surgit une écriture à partir de l'écriture primitive.

L'attention à la lettre, au signifiant, transforme le procédé classique de la lecture savante en surgissement d'une écriture nouvelle. Dans le texte, comme dans ce qu'on appelle l'expérience, l'important est moins le message, souvent évident, mais la modalité, échec de toute idéologie de la communication que cette dernière soit historique, théologique ou managériale. Devoir, pouvoir, savoir, croire, vouloir, jouent chez Surin (p. 242-244) comme chez Angelus Silesius (p. 279) un rôle d'altération et d'instauration : un « vouloir dire » (p. 344), comme le mettent en scène les glossolalies dans le balbutiant échec d'un dire.

Pour terminer, et pour livrer l'impression (au sens le plus fort du mot, de la marque qui s'imprime) que me laisse ce livre, je ferais une remarque personnelle, trace d'une lecture que l'énonciation présente en ces pages suscite en moi. Un procédé d'analyse, peut-être un artifice de lecteur rusé, mais confirmé par nombre d'expériences, s'est souvent imposé à moi, mais seulement à propos de livres qui sont de grands livres. C'est que le livre dit toujours en quelque endroit ce qu'il est et ce qu'il fait, il se dévoile lui-même en parlant d'un autre. J'ai ainsi été frappé en m’apercevant que ce que Michel de Certeau dit de Nicolas de Cues, en ces pages découvertes seulement aujourd'hui, peut s'appliquer à La Fable mystique, comme si la clef de ce livre était dissimulée en une place évidente, dans ce qu'il découvrait et exposait d'un autre. J'ai été frappé par la lecture faite par Michel de Certeau à la fois des textes (et ici au premier chef de la préface du De icona) et de l'expérience, mondaine, religieuse, philosophique, humaine, 
de Nicolas de Cues. Ce que Michel de Certeau en repère au premier abord, c'est ce qu'il appelle un "style ", " un style scientifique et théorique ", " une façon de faire », des "gestes [...] bien reconnaissables » (p. 60).

Au départ, chez l'auteur comme chez le lecteur, une admiratio, un étonnement, à la fois surprise et saisissement (p. 83, p. 119. Le terme est récurrent, c'est aussi celui de Georges de Trébizonde s'adressant au cusain !). L'admiratio suscite, ou rend possible, la pensée : la vision «permet de penser» (p. 102). Elle se diffracte pour ainsi dire et conduit l'œuvre du cusain à se produire « en des champs différents» (p. 60), en des langues différentes. Ces œuvres tracent «le présent d'un parler local dans un héritage reçu du passé » (p. 61). Je pourrais multiplier les citations qui nous incitent à estimer que ces pages sur Nicolas de Cues peuvent être dites les plus «autobiographiques» de Michel de Certeau; certes Michel de Certeau a toujours à juste titre estimé que le discours autobiographique était "impudique voire obscène ", comme il soulignait en 1972 lors du débat publié l'année suivante dans Crise du biblisme chance de la Bible. ${ }^{5}$ Mais c'est en parlant d'un autre, de l'œuvre d'un autre, qu'en creux le lecteur découvre cette dimension, l'admiratio pour le cusain et la justification de cette admiratio révélant peut-être un des secrets de l'œuvre de l'historien lui-même. Chez le penseur $d u X^{e}$ siècle comme chez celui du XXe , le passage entre les parlers manifeste un lien entre le plus ancien et le plus neuf, entre d'un côté le discours le plus ancien, voire archaïque, celui de la tradition théologique, celui des mystiques et celui de la folie et des glossolalies, et d'un autre côté celui des disciplines les plus "modernes ", la mathématique, la philosophie, chez le cusain, la linguistique, la sémiotique, la science des textes, la psychanalyse chez Michel de Certeau; chez l'un et chez l'autre un même passage entre des mondes, la même ouverture à des terres encore inexplorées.

Les remarques que me suggèrent les pages de Michel de Certeau sur Nicolas de Cues peuvent-elles avoir leur prolongement dans notre propre travail ? En effet l'admiratio suscitée à leur tour par ces pages de Michel de Certeau conduit le lecteur, et/ou celui qui travaille sur des sources semblables ou analogues, à envisager différemment l'objet, les modalités, en un mot le "style » de son propre travail. Si, selon Michel de Certeau, Nicolas de Cues n'a pas eu de « disciples » (p. 114), c'est que, tout en "saisissant », sa pensée " déroute. Jamais elle n'est où on la croit » (p. 114). Car cette pensée ne peut être séparée de son énonciation et une énonciation n'est ni réitérable ni transmissible. Il est peut-être trop tôt pour évaluer, en une analyse derechef historique et au-delà des inévitables références ou citations, la destinée et l'effectivité de la pensée et de l'écriture de l'histoire de Michel de Certeau ; cependant l'écart de la trentaine d'années qui sépare le tome II du tome I de La Fable mystique permet au moins, avec ce recul d' " une génération », de comparer la portée pour nous de ce tome II avec celle

5. Op. cit., p. 57. 
du tome I. Le saisissement, ou l'admiratio pour reprendre ce mot, qu'avait suscité en nous le tome I en 1982 a déjà marqué, d'une façon qui peut être inattendue, nombre de travaux qui ne font pas explicite référence à cette nouvelle écriture d'une histoire de la mystique et de la spiritualité. Le tome II ne saurait effectuer le même saisissement que produisit en sa nouveauté le tome I, mais il confirme, s'appliquant à des œuvres, des documents, des époques différents entre eux, qu'il s'agit moins de méthodes ou de leçons que de façons de faire et d'écrire qui n'exercent leur effet qu'en produisant d'autres façons de faire et d'écrire, d'autres énonciations.

Jacques LE BRUN

EPHE

jacleb@orange.fr 